\title{
Raça e o uso dos serviços de saúde bucal por idosos
}

\author{
Race and the use of dental health services by the elderly
}

Eliane Helena Alvim de Souza ${ }^{1}$

Pierre Andrade Pereira de Oliveira ${ }^{1}$

Ana Claudia Paegle ${ }^{1}$

Paulo Sávio Angeiras de Goes ${ }^{1}$

${ }^{1}$ Universidade de Pernambuco. Av Gal. Newton Cavalcanti 1650, Caixa Postal 1028 Camaragibe PE. e.ha.souza@hotmail.com
Abstract We analyze if race can be considered a limiting factor in the use of dental services by the elderly. This study is of an analytical nature, with the use of secondary data collected by the National Survey of Oral Health in 2003. Those examined who declared themselves as being white, brown or black in the 65 to 74-year-old age bracket were included. The sample was composed of 5,108 elderly people: 2,575 whites and 2,533 blacks. Of the whites, $3.8 \%$ stated that they had never been to the dentist, while this figure was $7.8 \%$ for the blacks. Even after the adjustment for interception for prosthetics and dental pain, the chance of elderly blacks not having used dental services at least once in their life is 0.62 OR less than for elderly whites. Of those who used the services, $21.2 \%$ of the elderly whites visited the dentist in the last year, while for elderly blacks the figure was 14.2\%, in the adjusted model for interception for prosthetics and dental pain the OR was 0.60. All the relations were statistically significant $(p<0,001)$. Race is a limiting factor in the use of dental services by the elderly and even after the adjustments the elderly blacks continue to manifest greater resistance to the use of oral health services.

Key words The elderly, Afro-descendants, Geriatric dentistry, Dental health services, Accessibility of health services
Resumo Analisamos se a raça pode ser considerada um fator limitante na utilização dos serviços odontológicos por idosos. O presente estudo é de caráter analítico, com a utilização de dados secundários coletados pela Pesquisa Nacional de Saúde Bucal em 2003. Foram incluidos os examinados que se declararam como brancos, pardos ou pretos, na faixa etária de 65 a 74 anos. O número amostral foi composto por 5.108 idosos sendo 2.575 brancos e 2.533 negros. Dos brancos 3,8\% afirmaram nunca terem ido ao dentista enquanto que para os negros esse valor é de 7,8\%. Mesmo após o ajuste com interceptação de necessidade de prótese e dor, a chance do idoso negro não ter utilizado o serviço odontológico pelo menos uma vez na vida é 0.62 OR menor que para um idoso branco. Dos que utilizaram os serviços, $21,2 \%$ dos idosos brancos visitaram o dentista no último ano, enquanto para os negros o valor foi de 14,2\%, no modelo ajustado interceptado pela necessidade de prótese e dor o OR foi de 0.60. Todas as relações foram estatisticamente significantes $(p<0,001)$. A raça é um fator limitante na utilização dos serviços odontológicos por idosos e, mesmo após ajustes, os idosos negros permanecem com maior dificuldade na utilização dos serviços de saúde bucal. Palavras-Chave Idoso, Negros, Odontologia geriátrica, Serviços de saúde bucal e acesso aos serviços de saúde 


\section{Introdução}

Poucos são os estudos que abordam a discussão entre as diferenças das condições de saúde e utilização dos serviços entre idosos e raça, mesmo estando diante de grupos fragilizados com necessidade crescente de evidencias no impacto do processo saúde-doença, afetados por limitações físicas, desigualdades e injustiças sociais.

Segundo a Organização Mundial de Saúde até 2025 o mundo terá cerca de $75 \%$ das pessoas com mais de 65 anos caracterizando um aumento na faixa etária e na expectativa de vida ${ }^{1}$. Com esse incremento da população idosa torna-se necessária a criação de serviços que proporcionem uma melhor qualidade de vida a essa população. A saúde bucal, como parte integrante da saúde geral deve merecer atenção especial. Há algum tempo, já existe a preocupação com a atenção à saúde bucal nas faixas etárias mais jovens, todavia é necessária a implantação de serviços de saúde integral também para os idosos². No Brasil, principalmente no serviço público, ainda são escassos os serviços especiais de atendimento odontológico ao idoso. A necessidade do desenvolvimento de uma política de promoção de saúde voltada para essa parcela da população é verificada em várias pesquisas que comprovam a carência desses serviços ${ }^{3-7}$.

Outra preocupação são as iniquidades raciais em saúde que vem ganhando espaço e são expressas pelos diferenciais nos riscos de adoecer e de morrer, originados de condições heterogêneas de existência e de acesso a bens e serviços. As diferenças são consideradas iníquas se decorrem do fato das pessoas disporem de escolhas limitadas, terem acesso restrito a recursos de saúde e estarem mais expostas a fatores prejudiciais. Diversos estudos documentam disparidades na saúde de grupos raciais distintos, em termos de morbimortalidade, e em relação ao acesso dos serviços de saúde $\mathrm{e}^{8-11}$. A utilização dos serviços de saúde bucal é um excelente indicador do modelo para compreensão das desigualdades sociais no acesso e condições de saúde entre raça e cuidados dentários $^{12}$, pois são razoavelmente de difícil acesso e alto custo. Portanto o objetivo desse estudo foi avaliar a relação da raça e o uso dos serviços de saúde bucal por idosos no Brasil.

\section{Metodologia}

O presente estudo é de caráter quantitativo, descritivo e analítico, com a utilização de dados se- cundários coletados pela Pesquisa Nacional de Saúde Bucal (SB Brasil) em $2003^{13}$. Foram incluídos apenas os examinados que se declararam como brancos, pardos ou pretos, na faixa etária de 65 a 74 anos.

O SB Brasil utilizou para coleta dos dados sobre o quesito "cor de pele", a classificação recomendada pelo IBGE $^{14}$ e pela Resolução 196/96 que estabelece normas de ética em pesquisa envolvendo seres Humanos ${ }^{15}$. Essa aferição tem como base a aparência física do individuo que responde ao questionamento do entrevistador "qual sua cor de pele/raça?", configurando a forma de autoclassificação que consiste na declaração do indivíduo sobre uma das cores/raça padronizadas: branco, parto, preto, amarelo ou indígena ${ }^{16}$.

A cor de pele é uma forma de classificação da raça utilizada no Brasil, ao juntarmos pardos e pretos está se falando de raça negra, ao ser usado separadamente está se falando de cor de pele ${ }^{17,18}$. O Estatuto da Igualdade Racial ${ }^{19}$ reafirma a população negra como o conjunto de pessoas que se autodeclaram pretas e pardas. Com base nessas recomendações que este estudo utilizou para classificação da variável raça a junção dos pardos e pretos formando a raça negra. Contudo o número amostral foi composto por 5.108 idosos sendo 2.575 brancos e 2.533 negros.

As variáveis utilizadas para verificar a utilização dos serviços odontológicos foram: se o entrevistado já visitou alguma vez na vida o dentista (não ou sim) e o tempo da última consulta (mais de um ano ou menos de um ano). Os agravos em saúde bucal utilizados para controlar o desfecho foram a necessidade de prótese superior, necessidade de prótese inferior e dor nos últimos seis meses.

Para resumo dos dados e análise utilizou-se o programa estatístico SPSS (Statiscal Package for Social Science). A análise bivariada foi usada para o estudo de associação entre variáveis no qual foi aplicado o teste estatístico qui-quadrado de independência. O modelo de análise multivariada através da regressão logística serviu para elucidar o quanto a raça pode explicar da limitação na utilização dos serviços odontológicos em idosos, sendo considerado um modelo ajustado em três fases, (1) sexo e idade, (2) sexo, idade, necessidade de prótese superior, inferior e dor nos últimos seis meses e (3) sexo, idade, necessidade de prótese superior, inferior ou dor nos últimos seis meses, renda e escolaridade.

As responsabilidades éticas quanto à realização dos exames foram atribuídas pelo projeto 
SB Brasil $2003^{13}$ que teve aprovação do Comitê de Ética em Pesquisa do Ministério da Saúde.

Com relação ao banco de dados do SB 2003, Queiroz et al. ${ }^{20}$ aponta que o processo de amostragem da pesquisa não foi finalizado, faltando o cálculo dos pesos amostrais necessários para a produção de estimativas válidas para a população brasileira. No entanto, o objetivo do presente estudo é avaliar a relação entre exposições (ex. raças dos idosos) e resultados (ex. utilização dos serviços e tempo da última consulta) e não produzir estimativas precisas para o país, a ausência de amostragem de pesos e variáveis estruturais nas análises não são de grande preocupação para testes de hipóteses ${ }^{16,21-23}$.

\section{Resultados}

Com a análise bivariada foi verificada a relação estatisticamente significante para os cruzamentos entre a utilização dos serviços odontológicos $\mathrm{x}$ raça, sexo, necessidade de prótese, renda e escolaridade. Os idosos negros que afirmaram nunca terem ido ao dentista na vida é mais que o dobro dos idosos brancos. A razão de prevalência entre a utilização do serviço por raça no modelo não ajustado mostra que a chance de um idoso negro usar o serviço é muito menor que a de um branco. Ajustados por sexo e idade a desigualdade é conservada. Ao acrescentar no ajuste a necessidade de prótese e dor o valor da razão de prevalência diminui, sendo o ajuste que mais impacta na redução das desigualdades entre raças, mesmo um idoso negro tendo apresentando um quadro de dor ou necessidade de prótese a chance de utilizar o serviço é menor que um idoso branco. Ao acrescentar a escolaridade e renda no modelo, o valor da razão de prevalência sofre uma nova redução, porém não suficiente para sanar as desigualdades entre as piores condições de utilização do serviço para idosos negros (Tabela 1).

Com relação ao tempo de utilização dos serviços na análise bivariada, foram estatisticamente significantes os cruzamentos entre tempo $\mathrm{x}$ raça, sexo, idade, dor, faixa etária e escolaridade. Os idosos brancos utilizaram mais os serviços odontológicos no último ano que os negros. $\mathrm{Na}$ análise sem ajuste a chance do idoso negro visitar o dentista no último ano permanece inferior comparada a do branco. Ajustado por sexo e idade o valor é um pouco reduzido; ao ser acrescentada ao modelo a necessidade de prótese e dor, a desigualdade permanece inalterada. Acumulando no ajuste a renda e escolaridade verifi- ca-se o maior impacto na diminuição da desigualdade, porém permanece menor a chance do idoso negro ter utilizado os serviços odontológicos no último ano (Tabela 2).

\section{Discussão}

Este estudo ratifica pesquisas anteriores que explicam as desigualdades nos indicadores de saúde entre a variável raça, remetendo aos determinantes sociais de saúde como único fator influente na pior condição de saúde para negros comparados aos brancos. Os resultados da presente pesquisa apontam a influência dos determinantes sociais como parte da explicação pelo pior acesso aos serviços de saúde bucal, porém os mesmos não conseguem explicar toda a diferença que podem ser atribuídas aos efeitos da discriminação.

Segundo Krieger $^{24}$ as desigualdades raciais em saúde como reflexo dos efeitos da discriminação podem ser medidas de forma indireta a partir da comparação através das diferenças nos resultados de saúde entre grupos raciais.

Os dados encontrados no presente estudo apontam a existência da limitação na utilização dos serviços odontológicos, a chance de um idoso negro entrar no Sistema de Saúde se apresenta muito inferior a de um idoso branco e mesmo conseguindo utilizar o serviço por pelo menos uma vez na vida, continua encontrando barreiras com relação ao tempo de utilização. Ainda que necessitando de tratamento, seja por necessidade de prótese ou por sentir dor de dente o idoso negro permanece encontrando maior dificuldade no acesso ao dentista; o fator raça continua sendo um fator limitante para idosos negros na utilização dos serviços odontológicos mesmo em casos de urgência onde a dor de dente em tese seria um potencializador na busca desses serviços.

Na literatura voltada para saúde bucal, poucos são os estudos que buscam elucidar essa relação apontando o preconceito e a discriminação e a variável raça. Indícios de preconceito ou discriminação por parte do cirurgião-dentista foi encontrada por Cabral et al. ${ }^{25}$ que apontou a raça do paciente como fator de decisão do cirurgiãodentista em extrair ou preservar um dente, indicando que o negro é submetido a mais extrações dentárias (25,5\% x 16,2\%) que o branco, mesmo estando nas mesmas condições clínicas.

Skaar e Hardie ${ }^{26}$ estudaram os fatores demográficos associados ao uso dos serviços odontológicos por idosos nos Estados Unidos e encontrou que os idosos de menor idade, com alta ren- 
Tabela 1. Modelo de análise multivariado da utilização dos serviços de saúde bucal interceptado por necessidade de prótese e dor entre raças da amostra de idosos do Brasil, SB 2003.

\begin{tabular}{|c|c|c|c|c|c|c|}
\hline \multirow[b]{2}{*}{ Variáveis } & \multicolumn{2}{|c|}{ Utilização* } & \multicolumn{2}{|c|}{ Não ajustado } & \multicolumn{2}{|c|}{ Ajustado por sexo e idade } \\
\hline & Não \% & Sim \% & $\mathrm{OR}(\mathrm{C} 1)$ & Valor P & $\mathrm{OR}(\mathrm{C} 1)$ & Valor $\mathbf{P}$ \\
\hline \multicolumn{7}{|l|}{ Raça } \\
\hline Branco & 3,8 & 96,2 & 1 & & 1 & \\
\hline Negro & 7,8 & 92,2 & $0.47(0.37-0.60)$ & $<0,001$ & $0.47(0.37-0.60)$ & $<0.001$ \\
\hline \multicolumn{7}{|l|}{ Sexo } \\
\hline Masculino & 6,9 & 93,1 & $0.73(0.58-0.91)$ & 0,006 & $0.73(0.58-0.93)$ & 0.010 \\
\hline Feminino & 5,1 & 94,9 & 1 & & 1 & \\
\hline \multicolumn{7}{|l|}{ Idade } \\
\hline 65 e 66 anos & 5,9 & 94,1 & $1.28(0.90-1.83)$ & 0,161 & $1.23(0.86-1.76)$ & 0,265 \\
\hline 67 e 68 anos & 4,6 & 95,4 & $1,02(0.76-1.36)$ & 0,894 & $0.98(0.73-1.33)$ & 0,919 \\
\hline 69 a 72 anos & 5,8 & 94,2 & $0.85(0.62-1.16)$ & 0,298 & $0.81(0.58-1.11)$ & 0,199 \\
\hline 73 e 74 anos & 6,9 & 93,1 & 1 & 0,196 & 1 & 0,210 \\
\hline \multicolumn{7}{|c|}{ Necessidade de Prótese Superior } \\
\hline Não & 2,8 & 97,2 & 1 & & & \\
\hline Sim & 11,9 & 88,1 & $0.21(0.16-1.27)$ & $<0,001$ & & \\
\hline \multicolumn{7}{|c|}{ Necessidade de Prótese Inferior } \\
\hline Não & 2,9 & 97,1 & 1 & & & \\
\hline Sim & 8,1 & 91,9 & $0.34(0.25-0.44)$ & $<0,001$ & & \\
\hline \multicolumn{7}{|l|}{ Dor } \\
\hline Sim & 4,8 & 95,2 & $1.27(0.95-1.71)$ & 0,106 & & \\
\hline Não & 6,1 & 93,9 & 1 & & & \\
\hline \multicolumn{7}{|l|}{ Faixa de Renda } \\
\hline Sem renda & 36,0 & 64,0 & $0.32(0.02-0.05)$ & $<0,001$ & & \\
\hline Até 1 salário & 6,8 & 93,2 & $0.24(0.16-0.37)$ & $<0,001$ & & \\
\hline De 1 a 2 salários & 5,2 & 94,8 & $0.32(0.21-0.49)$ & $<0,001$ & & \\
\hline Acima de 2 salários & 1,7 & 98,3 & 1 & $<0,001$ & & \\
\hline \multicolumn{7}{|l|}{ Escolaridade } \\
\hline Sem escolaridade & 8,2 & 91,8 & $0.10(0.03-0.32)$ & $<0,001$ & & \\
\hline 1 a 4 anos & 3,1 & 96,9 & $0.29(0.09-0.91)$ & 0,035 & & \\
\hline 5 a 8 anos & 9,0 & 91,0 & $0.09(0.02-0.30)$ & $<0,001$ & & \\
\hline 9 a 16 anos & 0,9 & 99,1 & 1 & $<0,001$ & & \\
\hline
\end{tabular}

da, brancos e com maior escolaridade tendem a apresentar maior uso dos serviços.

Martins et al. ${ }^{27}$ avaliaram o modelo de Anderson e Davidson ${ }^{28}$ investigando as características associadas ao uso de serviços odontológicos entre idosos dentados e edentados no sudeste do Brasil, usando como base os dados do SB2003. A análise multivariada mostrou adequação ao modelo apresentado, sendo a raça um dos fatores associados ao uso dos serviços de saúde bucal por idosos. O uso dos serviços odontológicos no último ano foi para os não-brancos dentados de $0,65(\mathrm{OR})$ e $0,34(\mathrm{OR})$ para os edentados.

No tocante às relações entre raça e saúde, em anos recentes esse objeto vem sendo alvo de um campo de reflexões e de intervenção política denominada saúde da população negra. Inserindo discussões sobre a forma de enfrentar o racismo à medida que parcelas significativas da sociedade reconhecem as desvantagens materiais e simbólicas sofridas pelos negros. Essas desvantagens tornam a variável raça um fator determinante de desigualdade social e de exposição social ao risco de adoecimento e morte ${ }^{29}$.

Tendo como base as grandes disparidades na utilização dos serviços de saúde bucal por idosos e grupos raciais apontadas nesse estudo e os objetivos da Política Nacional de Saúde da Pessoa Ido$\mathrm{sa}^{30}$ e a Política Nacional de Saúde Integral da População Negra ${ }^{31}$ sugere-se como medida afirmativa 
Tabela 1. continuação

\begin{tabular}{|c|c|c|c|c|}
\hline \multirow[b]{2}{*}{ Variáveis } & \multicolumn{2}{|c|}{$\begin{array}{l}\text { Ajustado por sexo, idade, } \\
\text { necessidade de prótese e dor }\end{array}$} & \multicolumn{2}{|c|}{$\begin{array}{l}\text { Ajustado por sexo, idade, necessidade } \\
\text { de prótese, dor, renda e necessidade }\end{array}$} \\
\hline & $\mathrm{OR}(\mathrm{C} 1)$ & Valor P & OR (C1) & Valor P \\
\hline \multicolumn{5}{|l|}{ Raça } \\
\hline Branco & 1 & & 1 & \\
\hline Negro & $0.62(0.48-0.80)$ & $<0,001$ & $0.71(0.53-0.94)$ & 0,019 \\
\hline \multicolumn{5}{|l|}{ Sexo } \\
\hline Masculino & $0.96(0.75-1.22)$ & 0,733 & $0.86(0.65-1.22)$ & 0,261 \\
\hline Feminino & 1 & & 1 & \\
\hline \multicolumn{5}{|l|}{ Idade } \\
\hline 65 e 66 anos & $1.17(0.81-1.69)$ & 0,407 & $1.07(0.71-1.59)$ & 0,754 \\
\hline 67 e 68 anos & $0.97(0.71-1.32)$ & 0,853 & $0.83(0.59-1.16)$ & 0,280 \\
\hline 69 a 72 anos & $0.87(0.62-1.21)$ & 0,400 & $0.25(0.55-1.16)$ & 0,246 \\
\hline 73 e 74 anos & 1 & 0,528 & 1 & 0,424 \\
\hline \multicolumn{5}{|c|}{ Necessidade de Prótese Superior } \\
\hline Não & 1 & & 1 & \\
\hline Sim & $0.26(0.19-0.36)$ & $<0,001$ & $0.25(0.17-1.37)$ & $<0,001$ \\
\hline \multicolumn{5}{|c|}{ Necessidade de Prótese Inferior } \\
\hline Não & 1 & & 1 & \\
\hline Sim & $0.87(0.59-1.26)$ & 0.463 & $0.93(0.62-1.41)$ & 0,744 \\
\hline \multicolumn{5}{|l|}{ Dor } \\
\hline Sim & $1.54(1.13-2.11)$ & 0,006 & $1.53(1.09-2.14)$ & 0,014 \\
\hline Não & 1 & & 1 & \\
\hline \multicolumn{5}{|l|}{ Faixa de Renda } \\
\hline Sem renda & & & $0.04(0.02-0.06)$ & $<0,001$ \\
\hline Até 1 salário & & & $0.35(0.22-0.55)$ & $<0,001$ \\
\hline De 1 a 2 salários & & & $0.44(0.27-0.70)$ & 0,001 \\
\hline Acima de 2 salários & & & 1 & $<0,001$ \\
\hline \multicolumn{5}{|l|}{ Escolaridade } \\
\hline Sem escolaridade & & & $0.18(0.05-0.60)$ & 0,005 \\
\hline 1 a 4 anos & & & $0.37(0.11-1.24)$ & 0,109 \\
\hline 5 a 8 anos & & & $0.14(0.04-0.47)$ & 0,001 \\
\hline 9 a 16 anos & & & 1 & $<0,001$ \\
\hline
\end{tabular}

* Todos significantes segundo o teste qui quadrado valor $\mathrm{p}<0,001$, exceto sexo $\mathrm{p}=0,006$, idade $\mathrm{p}=0,301$ e dor $\mathrm{p}=0,105$.

a criação de pactos entre as três esferas com metas pré-estabelecidas respeitando a realidade local no que se refere à facilitação da terapêutica e da reabilitação, sanando a necessidade por prótese e aliviando os casos de urgência por dor de dente, a criação de alternativas que garantam a entrada, $o$ acompanhamento e a permanência contínua desses grupos nos serviços odontológicos, assegurar os princípios antirracistas e não discriminatórios no processo de acolhimento pelos profissionais de saúde bucal tendo como principal eixo norteador as Equipes de Saúde da Família e os Centros de Especialidades Odontológica.

\section{Conclusões}

A partir dos dados obtidos concluiu-se que:

- Para os idosos a raça é um fator limitante na utilização dos serviços odontológicos.

- A chance de um idoso negro nunca ter ido ao dentista é maior que o dobro comparado a de um idoso branco.

- A chance do idoso negro ter utilizado os serviços de saúde bucal no último ano é menor que a de um idoso branco.

. O fato de necessitar de prótese ou apresentar dor não diminuiu a dificuldade de utilização do serviço pelo idoso negro. 
Tabela 2. Modelo de análise multivariado do tempo da última consulta com um dentista interceptado por necessidade de prótese e dor entre raças da amostra de idosos do Brasil, SB 2003.

\begin{tabular}{|c|c|c|c|c|c|c|}
\hline \multirow[b]{2}{*}{ Variáveis } & \multicolumn{2}{|c|}{$\begin{array}{l}\text { Tempo da última } \\
\text { consulta }\end{array}$} & \multicolumn{2}{|c|}{ Não ajustado } & \multicolumn{2}{|c|}{$\begin{array}{c}\text { Ajustado por sexo } \\
\text { e idade }\end{array}$} \\
\hline & $\begin{array}{c}\text { Mais de } 1 \\
\text { ano } \%\end{array}$ & $\begin{array}{c}\text { Menos de } \\
1 \text { ano \% }\end{array}$ & $\mathrm{OR}(\mathrm{C} 1)$ & Valor P & $\mathrm{OR}(\mathrm{C} 1)$ & Valor $\mathbf{P}$ \\
\hline \multicolumn{7}{|l|}{ Raça } \\
\hline Branco & 78,7 & 21,2 & 1 & & 1 & \\
\hline Negro & 85,8 & 14,2 & $0.62(0.53-0.72)$ & $<0,001$ & $0.60(0.51-0.70)$ & $<0.001$ \\
\hline \multicolumn{7}{|l|}{ Sexo } \\
\hline Masculino & 80,6 & 19,4 & $1.18(1.02-1.37)$ & 0,023 & $1.22(1.05-1.42)$ & 0.010 \\
\hline Feminino & 83,1 & 16,9 & 1 & & 1 & \\
\hline \multicolumn{7}{|l|}{ Idade } \\
\hline 65 e 66 anos & 79,8 & 20,2 & $1.28(0.90-1.83)$ & 0,001 & $0.87(0.71-1.07)$ & 0,001 \\
\hline 67 e 68 anos & 81,8 & 18,2 & $1,02(0.76-1.36)$ & 0,043 & $0.77(0.64-1.93)$ & 0,194 \\
\hline 69 a 72 anos & 83,1 & 16,9 & $0.85(0.62-1.16)$ & 0,161 & $0.64(0.51-0.79)$ & 0,006 \\
\hline 73 e 74 anos & 85,2 & 14,8 & 1 & 0,004 & 1 & $<0,001$ \\
\hline \multicolumn{7}{|c|}{ Necessidade de Prótese Superior } \\
\hline Não & 81,9 & 18,1 & 1 & & & \\
\hline Sim & 82,6 & 17,4 & $0.95(0.81-1.12)$ & 0,577 & & \\
\hline \multicolumn{7}{|c|}{ Necessidade de Prótese Inferior } \\
\hline Não & 82,1 & 17,9 & 1 & & & \\
\hline Sim & 82,2 & 17,8 & $0.92(0.86-1.15)$ & $<0,001$ & & \\
\hline \multicolumn{7}{|l|}{ Dor } \\
\hline Sim & 70,5 & 29,5 & $2.47(2.11-2.90)$ & $<0,001$ & & \\
\hline Não & 85,6 & 14,4 & 1 & & & \\
\hline \multicolumn{7}{|l|}{ Faixa de Renda } \\
\hline Sem renda & 69,9 & 30,1 & $1.30(0.88-1.90)$ & 0,183 & & \\
\hline Até 1 salário & 87,2 & 12,8 & $0.44(0.37-0.53)$ & $<0,001$ & & \\
\hline De 1 a 2 salários & 85,8 & 14,2 & $0.49(0.41-0.59)$ & $<0,001$ & & \\
\hline Acima de 2 salários & 75,0 & 25,0 & 1 & $<0,001$ & & \\
\hline \multicolumn{7}{|l|}{ Escolaridade } \\
\hline Sem escolaridade & 89,8 & 10,2 & $0.15(0.11-0.20)$ & $<0,001$ & & \\
\hline 1 a 4 anos & 82,6 & 17,4 & $0.28(0.22-0.35)$ & $<0,001$ & & \\
\hline 5 a 8 anos & 73,9 & 26,1 & $0.35(0.35-0.62)$ & $<0,001$ & & \\
\hline 9 a 16 anos & 56,8 & 43,2 & 1 & $<0,001$ & & \\
\hline
\end{tabular}

- Mesmo ajustado por sexo, idade, necessidade de prótese, dor, renda e escolaridade para o idoso negro é mais difícil ter assegurado o acesso aos serviços de saúde bucal que para os idosos não negros.

\section{Colaboradores}

EHA Souza, PAP Oliveira, AC Paegle e PSA Goes participaram igualmente de todas as etapas de elaboração do artigo. 
Tabela 2. continuação

\begin{tabular}{|c|c|c|c|c|}
\hline \multirow[b]{2}{*}{ Variáveis } & \multicolumn{2}{|c|}{$\begin{array}{l}\text { Ajustado por sexo, idade, } \\
\text { necessidade de prótese e dor }\end{array}$} & \multicolumn{2}{|c|}{$\begin{array}{l}\text { Ajustado por sexo, idade, necessidade } \\
\text { de prótese, dor, renda e necessidade }\end{array}$} \\
\hline & $\mathrm{OR}(\mathrm{C} 1)$ & Valor P & OR (C1) & Valor P \\
\hline \multicolumn{5}{|l|}{ Raça } \\
\hline Branco & 1 & & 1 & \\
\hline Negro & $0.60(0.51-0.70)$ & $<0,001$ & $0.73(0.62-0.86)$ & $<0,001$ \\
\hline \multicolumn{5}{|l|}{ Sexo } \\
\hline Masculino & $1.18(1.01-1.38)$ & 0,035 & $1.10(0.94-1.30)$ & 0,239 \\
\hline Feminino & 1 & & 1 & \\
\hline \multicolumn{5}{|l|}{ Idade } \\
\hline 65 e 66 anos & $0.84(0.68-1.04)$ & 0,106 & $0.83(0.66-1.03)$ & 0,051 \\
\hline 67 e 68 anos & $0.80(0.66-0.96)$ & 0,022 & $0.84(0.68-1.02)$ & 0,097 \\
\hline 69 a 72 anos & $0.65(0.52-0.82)$ & 0,400 & $0.73(0.58-0.93)$ & 0,078 \\
\hline 73 e 74 anos & 1 & $<0,001$ & 1 & 0,010 \\
\hline \multicolumn{5}{|c|}{ Necessidade de Prótese Superior } \\
\hline Não & 1 & & 1 & \\
\hline Sim & $0.94(0.77-1.16)$ & 0,588 & $1.12(0.91-1.38)$ & 0,297 \\
\hline \multicolumn{5}{|c|}{ Necessidade de Prótese Inferior } \\
\hline Não & 1 & & 1 & \\
\hline Sim & $0.84(0.59-1.21)$ & 0,903 & $1.04(0.86-1.25)$ & 0,673 \\
\hline \multicolumn{5}{|l|}{ Dor } \\
\hline Sim & $2.46(2.09-2.89)$ & $<0,001$ & $2.52(2.12-2.98)$ & $<0,001$ \\
\hline Não & 1 & & 1 & \\
\hline \multicolumn{5}{|l|}{ Faixa de Renda } \\
\hline Sem renda & & & $1.02(0.66-1.57)$ & $<0,001$ \\
\hline Até 1 salário & & & $0.58(0.47-0.72)$ & $<0,001$ \\
\hline De 1 a 2 salários & & & $0.64(0.53-0.78)$ & $<0,001$ \\
\hline Acima de 2 salários & & & 1 & \\
\hline \multicolumn{5}{|l|}{ Escolaridade } \\
\hline Sem escolaridade & & & $0.20(0.51-0.27)$ & $<0,001$ \\
\hline 1 a 4 anos & & & $0.33(0.25-0.43)$ & $<0,001$ \\
\hline 5 a 8 anos & & & $0.49(0.36-0.66)$ & $<0,001$ \\
\hline 9 a 16 anos & & & 1 & \\
\hline
\end{tabular}

* Todos significantes segundo o teste qui quadrado valor $\mathrm{p}<0,001$, exceto sexo $\mathrm{p}=0,023$, necessidade prótese superior $\mathrm{p}=0,577$, inferior $\mathrm{p}=0,922$.

\section{Referências}

1. Silvestre JA, Kalache A, Ramos LR, Veras RP. O envelhecimento populacional brasileiro e o setor de saúde. Arquivos de Geriatria e Gerontologia 1996; $0(1): 81-89$.

2. Souza EHA, Barbosa MBCB, Oliveira PAP, Espíndola J, Gonçalves KJ. Impacto da saúde bucal no cotidiano de idosos institucionalizados e não institucionalizados da cidade do Recife (PE, Brasil). Cien Saude Colet 2010; 15(6):2955-2964.

3. Meneghim MC, Pereira AC, Silva FRB. Prevalência de cárie radicular e condição periodontal em uma população idosa institucionalizada de PiracicabaSP. Pesqui Odontol Bras 2002; 16(1):50-56.
4. Shinkai RSA, Del Bel Cury AA. O papel da odontologia na equipe interdisciplinar: contribuindo para a atenção integral ao idoso. Cad Saude Publica 2000; 16(4):1099-1109.

5. Berti M. Autopercepção e avaliação das condições de saúde bucal de idosos [tese]. Araçatuba: Universidade Estadual Paulista Júlio de Mesquita Filho; 2002.

6. Silva DD, Souza MLR, Toledo R, Lisboa CM. Condições de saúde bucal em idosos na cidade de Piracicaba. Rev Assoc Paul Cir Dent 2002; 56(3):183-187.

7. Silva DD, Souza MLR, Wada RS. Saúde bucal em adultos e idosos na cidade de Rio Claro, São Paulo, Brasil. Cad Saude Publica 2004; 20(2):626-631. 
8. Barros FS, Meneghel SN, Olinto MTA. Citopatológico e exame de mama: desigualdade de acesso para mulheres negras no sul do Brasil. Epidemiol. Serv. Saúde 2008; 17(2):138-141.

9. Batista LE. Homens e mulheres negros: saúde, doença e morte [tese]. Araraquara, SP: Universidade Estadual Paulista; 2002.

10. Cruz ICF. Saúde e iniquidades raciais no Brasil: o caso da população negra. online Brazilian Journal of Nursing [on line] 2006; 5(2).

11. Pinheiro L, Fontoura NO, Querino AC, Bonetti A, Rosa W. Retrato das desigualdades de gênero e raça. $3^{\mathrm{a}}$ ed. Brasília: Ipea, SPM, Unifem; 2008.

12. Gilbert GH. Racial and socioeconomic disparities in health from population-based research to practice-based research: the example of oral health. $J$ Dent Educ 2005; 69(9):1003-1014.

13. Brasil. Ministério da Saúde. Secretaria de Atenção à Saúde. Departamento de Atenção Básica (DAB). Projeto SB Brasil 2003. Condições de saúde bucal da população brasileira, 2002-2003: resultados principais. Brasília: MS; 2004.

14. Instituto Brasileiro de Geografia e Estatística (IBGE). Manual do recenseador - CD 1.09. Rio de Janeiro: IBGE; 2000.

15. Brasil. Ministerio da Saúde. Resolução nº196/1996. Dispõe sobre pesquisa envolvendo seres humanos. Bioética 1996; 4(Supl. 2):5-25.

16. Bastos JL, Peres MA, Peres KG, Dumith SC, Gigante DP. Diferenças socioeconômicas entre autoclassificação e heteroclassificação de cor/raça. Rev Saude Publica 2008; 42(2):324-334.

17. Travassos C, Williams DR. The concept and measurement of race and their relationship to public health: a review focused on Brazil and the United States. Cad Saude Publica 2004; 20(3):660-678.

18. Oliveira F. Ser negro no Brasil: alcances e limites. Estud. av. 2004; 18(50):57-60

19. Brasil. Lei $n^{\circ} .12 .288$ de 20 de julho de 2010. Institui o Estatuto da Igualdade Racial; altera as Leis nos 7.716, de 5 de janeiro de 1989, 9.029, de 13 de abril de 1995, 7.347, de 24 de julho de 1985, e 10.778, de 24 de novembro de 2003. Diário Oficial da União 2010; 21 jul.

20. Queiroz RCS, Portela MC, Vasconcellos MTL. Pesquisa sobre as Condições de Saúde Bucal da População Brasileira (SB Brasil 2003): seus dados não produzem estimativas populacionais, mas há possibilidade de correção. Cad Saude Publica 2009; 25(1):47-58.
21. Narvai PC, Antunes JLF, Moysés SJ, Frazão P, Peres MA, Peres KG, Sousa MLR, Roncalli AG. Validade científica de conhecimento epidemiológico gerado com base no estudo Saúde Bucal Brasil 2003. Cad Saude Publica 2010; 26(4):647-670.

22. Moreira RS. Validade ou verdade? Algumas reflexões sobre crenças verdadeiras e justificáveis em epidemiologia. Cad Saude Publica. 2010; 26(4):663-665.

23. Barata RB. Epidemiologia vs. Estatística: a velha contenda entre racionalismo e empirismo? Cad Saude Publica 2010; 26(4):667.

24. Krieger N. Epidemiology, racism, and health: the case of low birth weight. Epidemiology 2000; 11(3):237-239.

25. Cabral ED, Caldas Jr AF, Cabral HAM. Influence of the patient's race on the dentist's decision to extract or retain a decayed tooth. Community Dent Oral Epidemiol 2005; 33(6):461-466.

26. Skaar DD, Hardie NA. Demographic factors associated with dental utilization among community dwelling elderly in the United States. J Public Health Dent. 2006; 66(1):67-71.

27. Martins AMEBL, Barreto SM, Pordeus IA. Características associadas ao uso de serviços odontológicos entre idosos dentados e edentados no Sudeste do Brasil: Projeto SB Brasil. Cad Saude Publica 2008; 24(1):81-92.

28. Andersen RM, Davidson PL. Ethnicity, aging, and oral health outcomes: a conceptual framework. $A d v$ Dent Res. 1997; 11(2):203-209.

29. Maio MC, Monteiro S, Chor D, Faerstein E, Lopes CS. Cor/raça no Estudo Pró-Saúde: resultados comparativos de dois métodos de auto-classificação no Rio de Janeiro. Cad Saude Publica 2005; 21(1):171180.

30. Brasil. Portaria no 1395. Dispõe sobre a Política Nacional de Saúde do Idoso. Diário Oficial da União 1999; $13 \mathrm{dez}$

31. Brasil. Ministério da Saúde (MS). Política nacional de saúde integral da população negra. Brasília: MS; 2007.

Artigo apresentado em 14/04/2011

Aprovado em 29/06/2011

Versão final apresentada em 09/07/2011 